\title{
Hippo pathway coactivators Yap and Taz are required to coordinate mammalian liver regeneration
}

\author{
Li Lu' ${ }^{1,2}$, Milton J Finegold ${ }^{3}$ and Randy L Johnson ${ }^{4}$
}

The mammalian liver has a remarkable capacity for repair following injury. Removal of up to two-third of liver mass results in a series of events that include extracellular matrix remodeling, coordinated hepatic cell cycle re-entry, restoration of liver mass and tissue remodeling to return the damaged liver to its normal state. Although there has been considerable advancement of our knowledge concerning the regenerative capacity of the mammalian liver, many outstanding questions remaining, such as: how does the regenerating liver stop proliferating when appropriate mass is restored and how do these mechanisms relate to normal regulation of organ size during development? Hippo pathway has been proposed to be central in mediating both events: organ size control during development and following regeneration. In this report, we examined the role of Yap and Taz, key components of the Hippo pathway in liver organ size regulation, both in the context of development and homeostasis. Our studies reveal that contrary to the current paradigms that Yap/Taz are not required for developmental regulation of liver size but are required for proper liver regeneration. In livers depleted of Yap and Taz, liver mass is elevated in neonates and adults. However, Yap/Taz-depleted livers exhibit profound defects in liver regeneration, including an inability to restore liver mass and to properly coordinate cell cycle entry. Taken together, our results highlight requirements for the Hippo pathway during liver regeneration and indicate that there are additional pathways that cooperate with Hippo signaling to control liver size during development and in the adult.

Experimental \& Molecular Medicine (2018) 50, e423; doi:10.1038/emm.2017.205; published online 5 January 2018

\section{INTRODUCTION}

How organ size is regulated in mammals during development and how tissue homeostasis is maintained in adults is a fundamental question that is relevant for normal organ function as well as in pathological situations such as cancer. Several theories have been put forth that address how organs achieve their normal size during embryogenesis, how they regulate their size proportionally with overall body size during neonatal and prepubescent growth, and how cell death and cell proliferation is balanced in adults to maintain tissue homeostasis and normal organ size. ${ }^{1}$ Although different theories differ among mechanisms that underlie organ size control and homeostasis, they all converge on fundamental cellular processes such as cell division and survival.

Hippo signaling has emerged as a key pathway in regulating mammalian organ size. ${ }^{2-4}$ First described in the fruit fly Drosophila, the Hippo signaling pathway has at its core a kinase cascade that functions to negatively regulate the activities of two key transcriptional coactivators, Yap and Taz. When the
Hippo signaling pathway is active, Yap and Taz are phosphorylated by the Lats1/2 kinases resulting in their destabilization and retention in the cytoplasm. Conversely, when the Hippo pathway is inactive, Yap and Taz enter the nucleus where they interact with a variety of transcription factors that control proliferation, survival and differentiation. Evidence that the Hippo signaling pathway is involved in mammalian organ size control was first obtained by examining the effect of hyperactivation of Yap. ${ }^{5,6}$ By expressing a mutant form of Yap that cannot respond to Hippo pathway inhibitory signals, it was shown that Yap can drive abnormal increases in organ size, most notably in the liver. Subsequently, these findings were extended by inactivation of upstream Hippo pathway components in a variety of tissues, thereby inducing endogenous Yap (and presumably Taz). These studies indicated that Hippo signaling was integral in organ size regulation in the mammalian liver $^{7-10}$ and heart. ${ }^{11}$ Taken together, these studies suggested that Hippo signaling acts as a link between organ

${ }^{1}$ Department of Genetics, University of Texas, MD Anderson Cancer Center, Houston, TX, USA; ${ }^{2}$ Baylor Genetics, Baylor College of Medicine, Houston, TX, USA; ${ }^{3}$ Department of Pathology and Immunology, Baylor College of Medicine, Texas Children's Hospital, Houston, TX, USA and ${ }^{4}$ Department of Cancer Biology, University of Texas, MD Anderson Cancer Center, Houston, TX, USA

Correspondence: Professor RL Johnson, Department of Cancer Biology, University of Texas, MD Anderson Cancer Center, 6767 Bertner Avenue, Houston, TX 77030, USA.

E-mail: rljohnso@mdanderson.org

Received 19 March 2017; accepted 11 May 2017 
size sensing mechanisms and regulation of cell survival and proliferation through modulating the activities of Yap and Taz.

Recently, it has become appreciated that the Hippo signaling pathway is not the only pathway that controls the activity of Yap and Taz: inputs from the cytoskeleton, extracellular matrix and direct interaction with molecules outside of the core Hippo signaling pathway such as angiomotin, have also been shown to regulate subcellular localization and transcriptional activities of Yap and Taz. ${ }^{12,13}$ These findings have broadened our current understanding of Yap and Taz regulation, and suggest alternative inputs into regulation of organ size via Yap/Taz. In addition, these studies highlight the complexity of Yap/Taz regulation at the molecular level and provide additional opportunity for understanding mechanisms that control mammalian organ size regulation.

Given the proposed importance of Yap and Taz in controlling mammalian organ size, we sought to directly determine their requirement using the mouse liver as a model system. To that end, we generated mice that lack both Yap and Taz in hepatocytes and biliary epithelial cells (Yap/Taz liver conditional knockout). Yap/Taz liver conditional knockout mice are viable and fertile and their liver to body weight ratio is enlarged. The enlarged livers of Yap/Taz mutants had significantly increased numbers of proliferating hepatocytes accompanied by indicators of liver injury, including elevated serum levels of the liver enzymes alanine transaminase (ALT) and aspartate transaminase (AST). Yap/Taz-mutant livers also regenerated less efficiently than wild-type controls following two-third partial hepatectomy (PHx). Yap/Taz depletion resulted in reduced numbers of hepatocytes incorporating bromodeoxyuridine (BrdU) and an inability to completely recover liver mass following two-third PHx. Taken together, our results indicate that Yap and Taz are not essential for achieving relatively normal liver to body weight ratios during normal development and in unstressed adults, however, they are required to mount efficient regenerative responses and for achieving complete restoration of liver mass following $\mathrm{PHx}$.

\section{MATERIALS AND METHODS}

\section{Generation and breeding of $y a f^{f l / f l}$ and $t a z^{f l f}$ mice}

Yap ${ }^{f l f l}$ and $t a z^{f l / f l}$ mice were previously described. ${ }^{14}$ These mice were bred to Albumin-cre mice, ${ }^{15}$ followed by backcrossing to homozygous-floxed animals to generate liver-specific deletion of these genes. Resulting mutants were labeled in this paper as $y a p^{\Delta} / t a z^{\Delta}$. The genetic background of all mice is C57BL/6. All mice were housed in MD Anderson conventional facility with a 12-h light/dark schedule and food and water supply. All procedures were approved by the University of Texas, MD Anderson Cancer Center Animal Care and Use Committee.

\section{Quantitative PCR}

Total mRNAs were extracted from liver tissues with TRIZOL reagent (Invitrogen, 15596-025) and purified by Qiagen RNeasy Mini Kit 74104. Quantitative RT-PCR analysis was carried out using One-Step TaqMan gene expression assays (Applied Biosystems, Foster City, CA, USA) according to the manufacturer's instructions. Assay IDs for yap and taz are Mm00494237 and Mm00513560, respectively.

\section{Two-third PHx}

Five- to eight-weeks-old mice were used for performing PHx. The procedure for two-third PHx has been previously described. ${ }^{16}$ Briefly, the mice were anaesthetized with and after opening the abdomen, the medium and left lobes were tied by silk suture to stop the blood low, followed by lobe resection. The abdomen was then sealed with silk sutures and the mouse was put on $37^{\circ} \mathrm{C}$ incubator for recovery. Mice were killed on $0 \mathrm{~h}(0 \mathrm{~h}), 6 \mathrm{~h}, 24 \mathrm{~h}, 48 \mathrm{~h}, 72 \mathrm{~h}$ and 7 days $(7 \mathrm{~d})$ after $\mathrm{PHx}$ and the livers were harvested for protein extractions and paraffin sections. Fourteen-day time point was done only for measurement of liver/body weight ratio.

\section{Immunostaining}

Liver tissues were fixed in $4 \%$ PFA overnight at $4{ }^{\circ} \mathrm{C}$ and processed for paraffin embedding. Paraffin sections were cut at $5 \mu \mathrm{m}$. For CK19 staining, $10 \mathrm{~mm}$ sodium citrate $(\mathrm{pH} 6)$ was used for antigen retrieval. After serum blocking, tissue sections were incubated with primary antibody cytokeratin19 (CK19, rabbit, gift from Texas Children's Hospital Dr Milton Finegold's lab) at $4{ }^{\circ} \mathrm{C}$ overnight. Next day after phosphate-buffered solution wash, sections were incubated with fluor488 secondary antibody. Cell nuclei were stained with DAPI. Tissue sections were sealed and imaged with confocal microscopy. For labeling of cells undergoing DNA synthesis, $0.01 \mathrm{ml} \mathrm{g}^{-1}$ body weight of a $3 \mathrm{mg} \mathrm{ml}^{-1}$ solution of BrdU (Sigma-Aldrich, St Louis, MO, USA, B9285) in phosphate-buffered solution was injected IP $2 \mathrm{~h}$ before killing the mice. BrdU staining was carried out using the BrdU In-Situ Detection Kit (BD Biosciences Pharminigen, San Diego, CA, USA, 550803). For quantification of BrdU-positive hepatocytes, five different areas in each sample were photographed and counted. The result was statistically analyzed by one-way Anova.

\section{Protein extraction and analysis}

Total protein was extracted by RIPA buffer with present of both protease inhibitor (Roche Molecular Systems, Inc., Pleasanton, CA, USA, 04693132001) and phosphatase inhibitor (Roche Molecular Systems, Inc., 04906837001). Cytoplasmic and nuclear portion of protein were extracted by NE-PER Nuclear and Cytoplasmic Extraction Reagents (Thermo Fisher Scientific, Waltham, MA, USA, \#78835). Protein concentration was measured with BCA Protein Assay Reagent (Thermo Fisher Scientific, 23227).

\section{Western blot}

Protein samples were denatured by being boiled for $5 \mathrm{~min}$ with protein-loading buffer containing 5\% beta-metheltransferase. Western blots were run on $10 \%$ acrylamide gels followed by semi-dry polyvinylidene fluoride (PDVF) membrane transfer. PVD membranes were then blocked in 5\% milk in tris buffered saline/tween 20 (TBST) for $1 \mathrm{~h}$ at room temperature followed by primary antibody incubation in $5 \%$ bovine serum albumin (BSA) at $4{ }^{\circ} \mathrm{C}$ overnight. Secondary antibody was incubated at room temperature for $30 \mathrm{~min}$. After wash, the membranes were developed by enhanced chemiluminescence (ECL, Perkin Elmer, Waltham, MA, USA, NEL103001EA), and signals were detected by X-ray film. Primary antibodies used were phosphoYap/Taz (1:500, Cell Signaling Technology, Danvers, MA, USA, \#4911), Yap (1:500, Cell Signaling \#4912), Taz (1:1000, BD Biosciences Pharminigen \#560235), phospho-Mst1/2 (1:1000, Cell Signaling Technology \#3681), phospho-Lats1/2 (1:1000, Cell Signaling Technology \#9159), $\beta$-Actin (1:50000, Abcam, Cambridge, MA, USA, ab49900), CyclinD1 (1:1000, Cell Signaling Technology \#2922) and PCNA (1:1000, Cell Signaling Technology \#2586). 


\section{RESULTS}

Efficient deletion of Yap and Taz in the mouse liver

To generate mice that lack Yap and Taz in the biliary epithelial cell and hepatocyte lineages of the liver, we crossed conditional alleles of Yap and $\mathrm{Taz}^{14}$ to mice that contain an Albumin-cre transgene that directs cre-mediated recombination in fetal hepatic progenitor cells and in adult hepatocytes. ${ }^{15}$ To confirm depletion of Yap and Taz in the liver, we performed qRT-PCR and western analysis on extracts from wild-type and age-
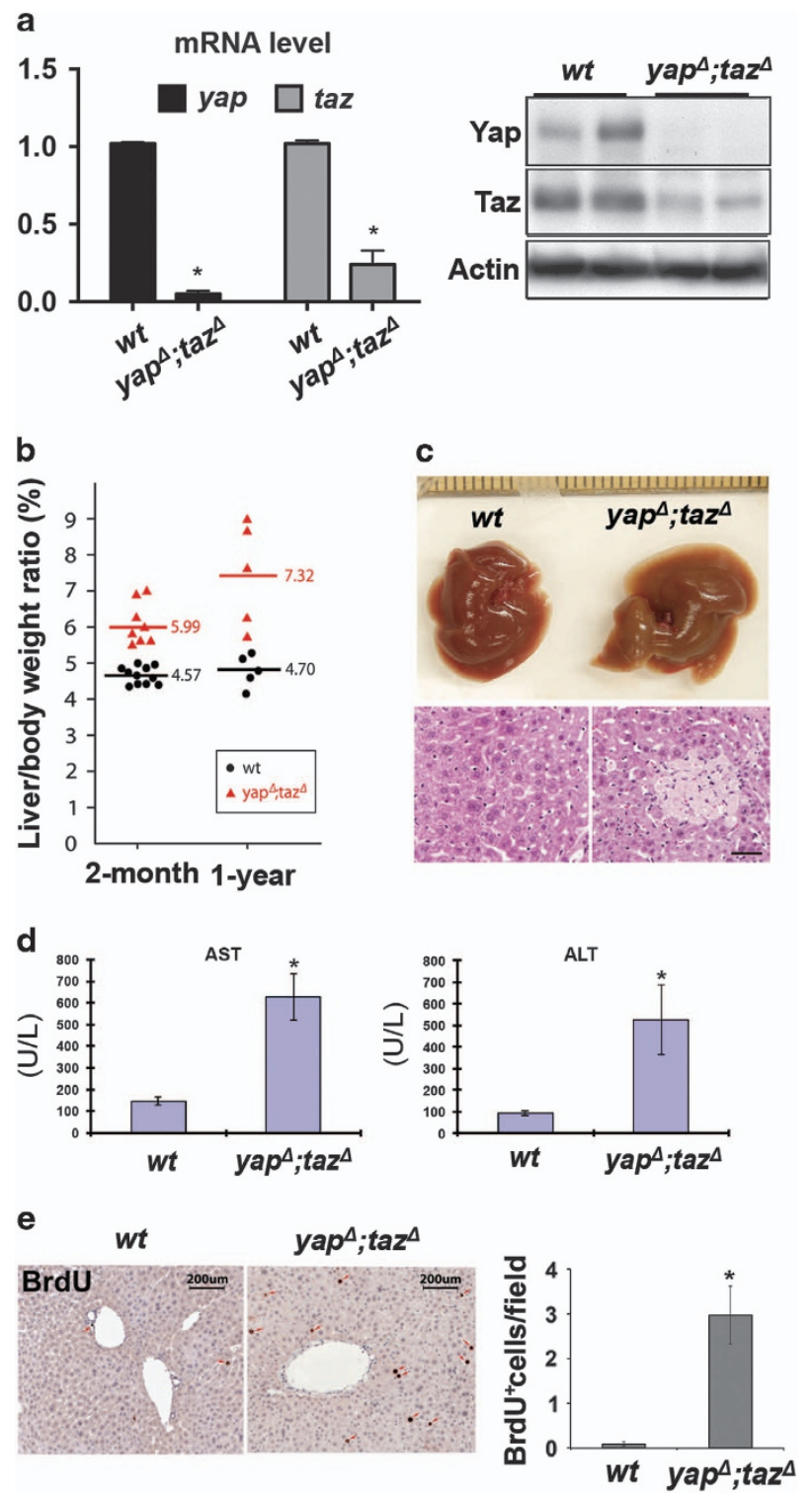

Figure 1 Efficient deletion of Yap and Taz in the mouse liver. (a) Both mRNA and protein levels of the Yap and Taz are significantly reduced in $y a p^{\Delta} /$ taz $^{\Delta}$ liver. (b) $Y_{a p^{\Delta}} /$ taz $^{\Delta}$ liver is $\sim 20 \%$ larger than wild-type (wt) counterparts at 2 month old and $\sim 50 \%$ larger at 1 year old. (c) Hepatic macrophages in area of necrosis in 2-month-old $\mathrm{yap}^{\Delta} / \mathrm{taz}^{\Delta}$ liver suggests hepatic injury. (d) Elevated serum AST and ALT levels in 2-month-old yap $\mathrm{p}^{\Delta} /$ taz $^{\Delta}$ liver indicate hepatic injury by yap/taz deletion. $N=3$. (e) Compensatory hepatic proliferation evidenced by significantly increased BrdU incorporation in 2-month-old $y a p^{\Delta} /$ taz $^{\Delta}$ liver. $N=3$. ${ }^{*} P<0.05$. matched Yap/Taz mutants $\left(y a p^{\Delta} / t a z^{\Delta}\right)$ (Figure 1a). Both Yap and Taz levels were efficiently reduced in $y a p^{\Delta} / t a z^{\Delta}$ livers as evidenced by significantly diminished RNA and protein levels.

Hepatomegaly, liver injury and compensatory proliferation in $\operatorname{yap}^{\Delta} /$ taz $^{\Delta}$ mutants

To examine the consequences of Yap and Taz depletion on liver size, we killed mice at different time points, harvested their livers and compared liver to body weight ratios. At 2 months of age, $y a p^{\Delta} /$ taz $^{\Delta}$ livers were significantly larger (20\%) then their wild-type counterparts. Liver enlargement was also observed at 1 year of age when $y a p^{\Delta} / t_{a z} z^{\Delta}$ livers had increased in size to $50 \%$ larger than their wild-type counterparts (Figure 1b). Histologically, yap $p^{\Delta} /$ taz $^{\Delta}$ livers exhibited signs of hepatic macrophages in area of necrosis suggestive of hepatocyte injury (Figure 1c). To investigate this observation further, we first compared serum levels of AST and ALT (Figure 1d) in 2-month-old $y a p^{\Delta} /$ taz $^{\Delta}$ mutants relative to control mice. Indeed, $y a p^{\Delta} / t_{a} z^{\Delta}$-mutant mice had significantly elevated serum AST and ALT levels indicating liver injury in these mice. Liver injury is frequently accompanied by compensatory hepatocyte proliferation. To determine whether yap ${ }^{\Delta} /$ taz $^{\Delta}$ mutants have increased rates of hepatocyte cell cycle entry, we pulsed mice with BrdU and quantified numbers of hepatocytes that incorporated BrdU in their nuclei, indicating entry into S-phase. Compared with the wild-type controls, yap ${ }^{\Delta} /$ taz $^{\Delta}$-mutant mice had significantly elevated numbers of BrdU incorporating hepatocytes (Figure 1e), suggesting that compensatory proliferation occurs upon Yap/Taz depletion.

Inflammation, biliary tract defects and adenoma formation in $y a p^{\Delta} / \operatorname{taz}^{\Delta}$-mutant livers

Albumin-cre is active in fetal hepatocytes, the common progenitor to biliary epithelial cells and hepatocytes in the adult. ${ }^{15}$ To examine the consequences of Yap/Taz depletion on the biliary epithelial cell lineage, we examined bile ducts of wild-type and $y a p^{\Delta} / \operatorname{taz}^{\Delta}$ mice by histology (Figure 2a) and immunostaining (Figure 2b). Inflammation around the bile ducts is found in $y a p^{\Delta} / t a z^{\Delta}$-mutant livers (Figure 2a). Wellformed bile ducts were observed in periportal regions of wildtype mice that were CK19-positive; in contrast, bile ducts in yap ${ }^{\Delta} /$ taz $^{\Delta}$-mutant livers are irregularly shaped and less wellformed in comparison (Figure 2b). Unexpectedly, at 1 year of age $y a p^{\Delta} /$ ta $^{\Delta}$-mutant mice developed liver adenomas (Figure $2 \mathrm{c}$ and $\mathrm{d}$ ). We did not observe other liver tumors in these mice at 1 year of age, including hepatocellular carcinoma or cholangiocarcinoma.

\section{Regulation of Hippo signaling during liver regeneration}

It has previously been reported that Yap and Taz activities are modulated following liver injury, including after $\mathrm{PHx} .{ }^{17}$ To confirm and extend these findings, we examined the phosphorylation status of Yap, Taz and upstream components at different time points after PHx. Consistent with previous findings, we observe that both Yap and Taz phosphorylation transiently decrease after PHx (Figure 3a and b), suggesting 


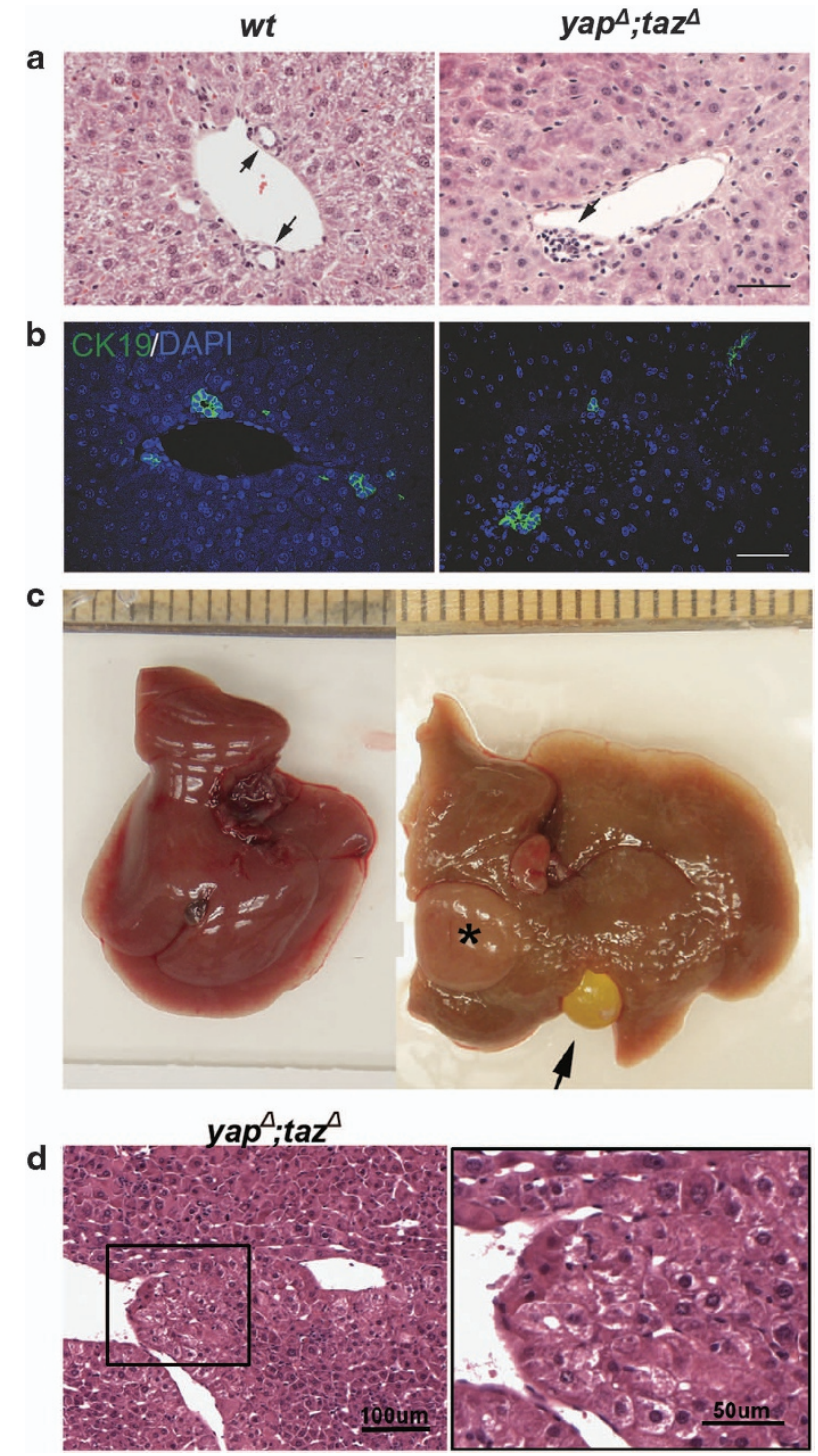

Figure 2 Inflammation, biliary tract defects and adenoma formation in $y a p^{\Delta} /$ taz $z^{\Delta}$ livers. (a) Inflammation around bile ducts in $y a p^{\Delta} /$ taz $^{\Delta}$ livers seen by H\&E histology. Scale bar is $50 \mu \mathrm{m}$. (b) The irregularly shaped and less well-formed bile ducts in $y a p^{\Delta} /$ taz $^{\Delta}$ livers distinguished by CK19 staining. Scale bar is $50 \mu \mathrm{m}$. (c) Gross view of a 1 year old wild-type liver and a $y a p^{\Delta} /$ taz $^{\Delta}$ liver with adenoma (star labeled) and enlarged gallbladder (arrows pointed). (d) The liver adenoma histology of low-power (left) and high-power (right) in 1 year old yap $^{\Delta} /$ taz $^{\Delta}$ liver.

that they become activated in response to PHx. To investigate this observation further, we assayed nuclear and cytoplasmic localization of Yap during PHx by western analysis (Figure 3c). Prior to surgery, Yap is predominantly cytoplasmic, however, at $6 \mathrm{~h}$ post-hepatectomy, equal levels of Yap are seen in nuclear and cytoplasmic fractions, indicative of elevated Yap activities. Elevated nuclear levels of Yap are also observed at 24, 48 and $72 \mathrm{~h}$ post-hepatectomy although the relative levels of nuclear Yap decrease due to increased cytoplasmic Yap localization during this period. In contrast to previous reports, ${ }^{17,18}$ we did not observe marked alterations in phosphorylation of upstream Hippo pathway components Mst1/2 or Lats1/2 (Figure 3d), although our analysis may not detect changes in their activities that are mediated by phosphorylation at residues not detected by the antibody reagents used in our study or by nonphosphorylation-mediated mechanisms.

\section{Inefficient liver regeneration in $y a p^{\Delta} /$ taz $^{\Delta}$ mice}

To assess the requirement(s) for Yap and Taz in controlling compensatory proliferation following $\mathrm{PHx}$, we performed twothird PHx in control and $y a p^{\Delta} / t a z^{\Delta}$ mice. Liver regrowth was blunted in $y a p^{\Delta} /$ taz $^{\Delta}$ mice at all time points after $\mathrm{PHx}$, including at 1 and 2 weeks after surgery, time points when wild-type mice had completely regained liver mass (Figure 4a). This defect in restoration of liver mass was paralleled by a diminished entry of hepatocytes into S-phase as assayed by BrdU incorporation (Figure $4 \mathrm{~b}$ and c). At $48 \mathrm{~h}$, where BrdU incorporation in wild-type livers is maximal, $y a p^{\Delta} / \operatorname{taz}^{\Delta}$ livers have significantly fewer hepatocytes that incorporate BrdU. This effect is also observed at $72 \mathrm{~h}$ post PHx. At 7 days after surgery, BrdU incorporation has returned to baseline levels in both wild-type and $y a p^{\Delta} / \operatorname{taz}^{\Delta}$ mutants but remains significantly higher in $y a p^{\Delta} /$ taz $^{\Delta}$ mutants, consistent with their elevated levels prior to surgery. Defects in BrdU incorporation in $y a p^{\Delta} /$ taz ${ }^{\Delta}$ mice are mirrored by defects of cell cycle progression marked by levels of PCNA and cyclinD1 (Figure 4d). In wildtype mice, PCNA and cyclinD1 are markedly induced following PHx with peak levels seen at 48 and $24 \mathrm{~h}$, respectively. In contrast, $y a p^{\Delta} /$ taz $^{\Delta}$-mutant mice show markedly elevated baseline levels of cyclinD1 and reduced induction of both cyclinD1 and PCNA after PHx. Taken together, these results indicate that although Yap/Taz are not absolutely required for liver regeneration, they are required for efficient cell cycle entry and for complete restoration of liver size following $\mathrm{PHx}$.

\section{DISCUSSION}

The Hippo pathway and its coactivators Yap and Taz have been implicated in regulating organ size, tissue regeneration and are activated in a wide variety of solid tumors. In this study, we have examined the role of Yap and Taz in controlling mammalian liver size during development and in the perinatal period. Our findings indicate that Yap/Taz are not obligate regulators of hepatocyte proliferation in the embryo or adult. In fact, $y a p^{\Delta} /$ taz $^{\Delta}$ livers are larger relative to their wild-type counterparts. In contrast, during liver regeneration, $y a p^{\Delta} / t z^{\Delta}$ mutants are defective in liver regrowth relative to their wildtype counterparts indicating an essential role for Yap and Taz in mediating responses to acute liver injury that require compensatory proliferation. The implications of this study on our current understanding of Hippo pathway in control of mammalian organ size, homeostasis, regeneration and disease are discussed below.

\section{Organ size control}

Activation of Yap, either by expression of a mutant form of Yap that is not effectively inhibited by Hippo pathway 
a

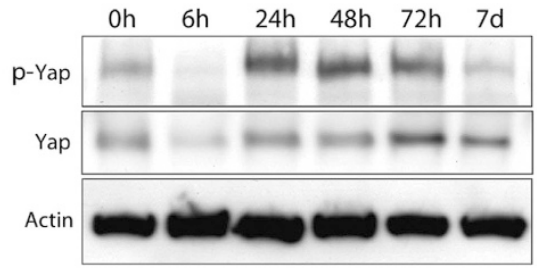

b

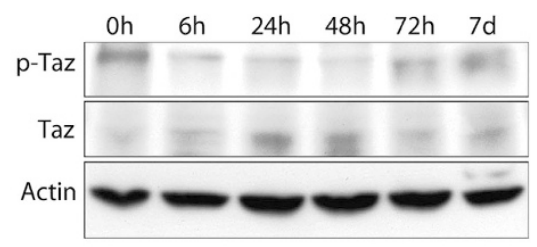

C

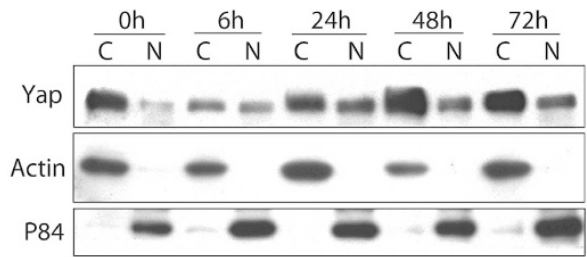

d

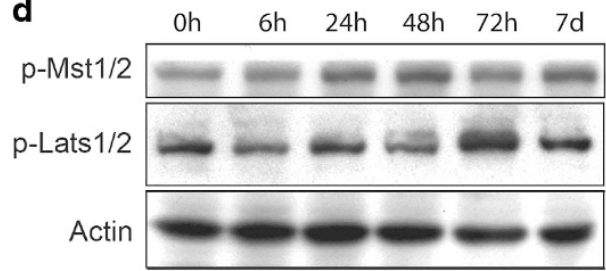

Figure 3 Regulation of Hippo signaling during liver regeneration. (a) Yap phosphorylation transiently decreases after partial hepatectomy ( $\mathrm{PHx})$, suggesting Yap activation during liver regeneration. (b) Taz phosphorylation also decreases after $\mathrm{PHx}$, suggesting Taz activation during liver regeneration. (c) Nuclear and cytoplasmic localization of Yap after PHx by western analysis. (d) Phosphorylation activations of Mst1/2 and Lats $1 / 2$ after PHx are not seen. Results were confirmed in three independently repeated experiments.

kinases, ${ }^{5,6}$ or by deletion of the Hippo pathway components, NF2, Sav1, Mst1/2 and Lats1/2, ${ }^{7-11,19-21}$ has profound effects on organ size. For example, in both the heart and liver, activation of Yap by these means results in increased organ size, both in the embryo and adult. These observations have led to the proposal that the Hippo pathway is dynamically regulated during embryogenesis and during perinatal periods to control Yap (and Taz) activities. ${ }^{22}$ According to these models, as yet undermined organ size control signals impact Yap/Taz activities through the Hippo signaling pathway. When an organ has not yet achieved its proper size, Hippo pathway is attenuated, thereby allowing for Yap/Taz-mediated proliferation to ensue. When proper organ size has been achieved, Hippo pathway activity is enhanced resulting in inhibition of Yap/Taz and stopping organ growth. Although this model has received support from transgenic and knockout studies mentioned above that activate Yap and Taz, relatively little is known about whether Yap and Taz are required to achieve proper organ sizes in neonates and in the perinatal period. Several studies have investigated this question by deleting Yap, for example, in the heart and liver, and current data suggest that Yap is not required for organ size control in these tissues. One potential explanation for these observations is that loss of Yap is compensated for by the maintained presence of Taz in these experiments. Accordingly, depletion of Taz in the context of Yap knockout would result in defects in organ size with the prediction that organs cannot grow to their proper sizes in the combined absence of Yap and Taz. Our results clearly indicate that this is not the case, at least for the mammalian liver. Hence, other mechanism(s) must be operating that mask an essential requirement for Yap/Taz in organ size control or that Yap/Taz are not integral components of pathways that control organ size in embryos or in pre-adult growth stages.

\section{Organ homeostasis}

In adults, organ size is maintained by a balance of cell proliferation and cell death. ${ }^{1}$ In some tissues such as the skin and intestine, stem cells fuel tissue renewal through production of transient amplifying cells that differentiate into mature cells of the skin and intestine. This rapid cell growth is balanced equally by cell death to maintain organ size. In other tissues such as the heart and liver, cell division is normally kept at very low levels. In contrast to the normal liver, we observe highly elevated BrdU incorporation rates in $y a p^{\Delta} / t_{a z}{ }^{\Delta}$ livers, which was also previously observed in Yap-mutant liver tissues. ${ }^{23}$ This increase in hepatocyte proliferation likely contributes to the increased liver/body weight ratio in $y a p^{\Delta} /$ taz $^{\Delta}$ mutants. As with the Yap mutants, $y a p^{\Delta} / t a z^{\Delta}$ livers have defective biliary structures that most likely result in accumulation of toxic bile acids that result in liver injury and hepatocyte cell death that trigger compensatory proliferation. Indeed, we observe histological signs of hepatocyte macrophages in area of necrosis and elevated serum AST and ALT levels in Yap/Taz-mutant mice. These observations indicate that Yap/Taz most likely affect liver homeostasis indirectly through defects in the biliary epithelial cell development.

\section{Organ regeneration}

The Hippo signaling pathway has been shown to be important in regulating regeneration of several tissues, including the intestine ${ }^{24}$ and the heart. ${ }^{11}$ In the intestine, Yap is activated in response to injury and is required for intestinal regeneration. In the heart, Hippo signaling acts as a barrier for regeneration. In mice, normal cardiomyocytes lose the ability to mount a regenerative response within a week after birth and that is accompanied by elevated Hippo signaling and reduced Yap/Taz activation. Relieving this inhibition restores the ability of cardiomyocytes to regenerate and to repair cardiac injury. In the liver, previous studies have implicated Hippo signaling in compensatory proliferation after $\mathrm{PHx},{ }^{17}$ but direct evaluation of the role of Yap and Taz in this process has not been demonstrated. Here we have shown that Yap/Taz are indeed required for normal liver regeneration, but not absolutely essential. Yap ${ }^{\Delta} /$ taz $^{\Delta}$-mutant hepatocytes can participate in a 


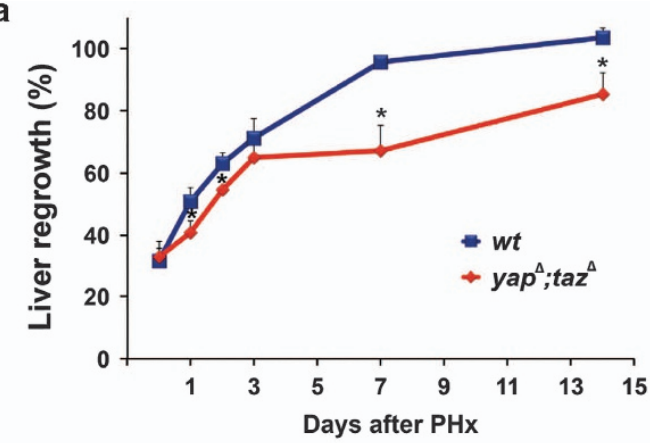

b

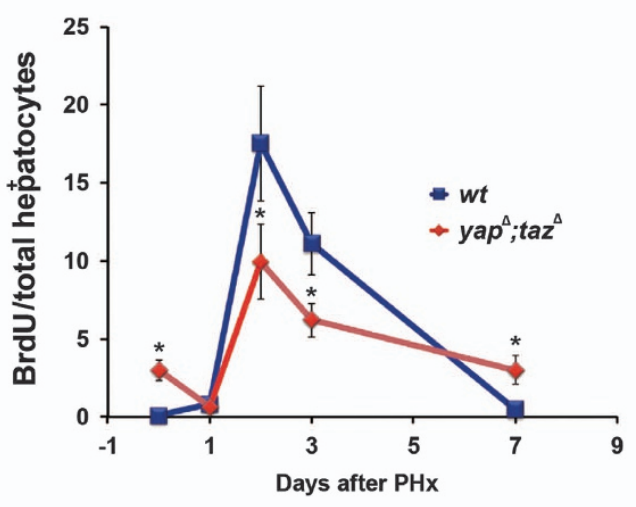

C

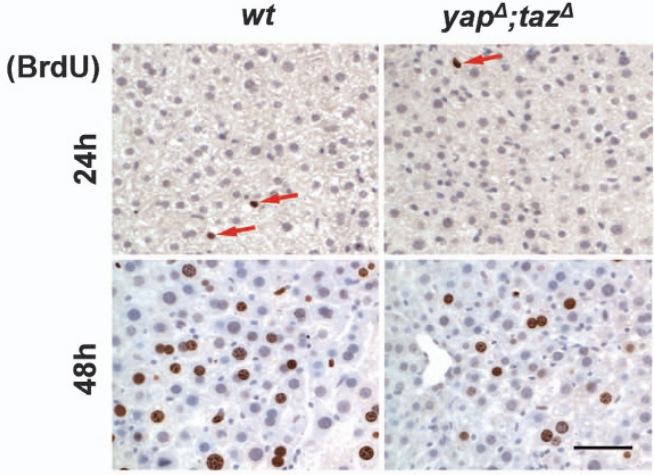

d

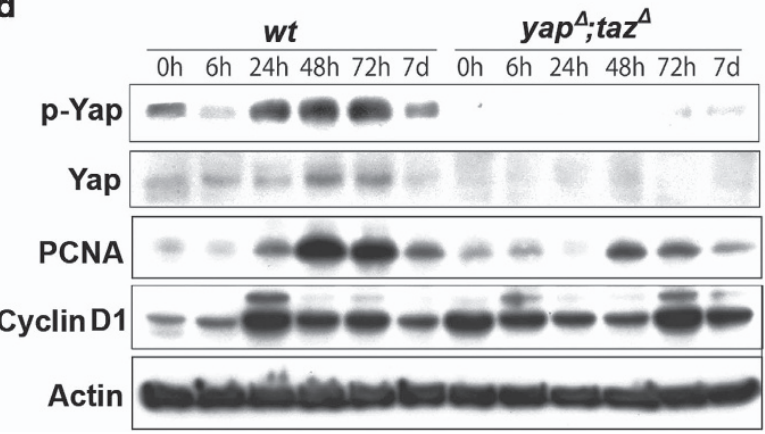

Figure 4 Inefficient liver regeneration in $y a p^{\Delta} / \operatorname{taz}^{\Delta}$ mice. (a) Liver regrowth is blunted in $y a p^{\Delta} /$ taz $^{\Delta}$ liver at all time points. (b) Diminished entry of $y a p^{\Delta} / t_{a} z^{\Delta}$ hepatocytes in to S-phase as assayed by BrdU incorporation. (c) Represented images of BrdU staining at 24 and $48 \mathrm{~h}$ after partial hepatectomy. Scale bar is $50 \mu \mathrm{m}$. (d) Deficient hepatic cell cycle re-entry and progression in yap $\mathrm{p}^{\Delta}$ taz $^{\Delta}$ liver by western analysis of the cell cycle markers. ${ }^{*} P<0.05$. Results were confirmed in three independently repeated experiments. regenerative response, albeit not as efficiently as wild-type hepatocytes. Whether this defective regeneration is a direct result of Yap/Taz inactivation in hepatocytes or is an indirect result of defects in biliary epithelial cells is unclear at this time. Additional experiments, such as deletion of Yap/Taz selectively in adult hepatocytes will be required to address this question.

That $y a p^{\Delta} /$ taz $^{\Delta}$ livers can mount a regenerative response results suggest that other pathways such as growth factor activation may partially compensate for Yap/Taz loss in the regenerating liver. Our results and those of others ${ }^{17}$ have clearly shown that Yap/Taz phosphorylation and cytoplasmic/nuclear localization is dynamically regulated during liver regeneration. Mechanistically, it is unclear how Yap and Taz are regulated during this process. Previous studies suggested that alterations in the kinase activity of upstream Hippo pathway components contribute to Yap/Taz activation. Indeed, genetic and/or pharmacological inhibition of Yap/Taz by affecting Mst1/2 kinase activities has been shown to augment liver regeneration. ${ }^{18,25}$ For reasons that are not clear at this time, we did not observe significant alterations in the Hippo pathway components Mst1/2 or Lats1/2, suggesting that other pathways are regulating Yap/Taz subcellular localization in this context. It may be possible that other kinases besides Mst1/2 or Lats1/2 are required to inhibit Yap/Taz during liver regeneration and there is some evidence from other systems that this may be the case. ${ }^{26}$ In addition, it is well appreciated that an early step in liver regeneration is remodeling of the extracellular matrix. ${ }^{27}$ As matrix composition and stiffness is a well-known regulator of Yap/Taz nuclear localization and activity, ${ }^{12}$ one attractive model is that matrix remodeling induces changes in Yap/Taz activity during early stages of liver regeneration. Further experiments are required to address this question.

\section{Liver disease}

Activation of Yap/Taz in the mouse liver results in liver injury and eventually formation of liver cancer. ${ }^{5-7}$ In addition, Yap and/or Taz activation occurs from bile acid-induced liver injury and in non-alcoholic hepatosteatosis. ${ }^{23,28,29}$ These and related findings suggest that Yap/Taz activation is a common event in liver disease and that sustained Yap/Taz activation contribute to disease progression to liver cancer. Indeed, several studies have shown that Yap inhibition delays disease progression in genetically engineered mouse models of hepatocellular carcinoma ${ }^{30}$ and that Yap inhibition is effective in treating advanced hepatocellular carcinoma. ${ }^{31}$ Our observation that $y a p^{\Delta} / \operatorname{taz}^{\Delta}$ mutants develop liver adenomas is somewhat paradoxical given previous findings that implicate Yap/Taz as oncogenes in hepatocarcinogenesis. A plausible explanation for this observation is that defects in the biliary system of $y a p^{\Delta}$ / $t a z^{\Delta}$ mutants leads to chronic liver injury setting the stage for liver cancer formation independently of Yap/Taz activation. There is evidence that in human hepatocellular carcinoma $\sim 20-30 \%$ of patients have a gene expression signature that suggests activation of Yap/Taz..$^{31,32}$ Similarly, immunohistochemical staining for Yap levels and localization have 
demonstrated that $20-40 \%$ of human hepatocellular carcinomas have high levels and nuclear localization of Yap (reviewed in ref. 33 However, these studies also suggest that hepatocellular carcinoma can develop without Yap and/or Taz activation. Another frequent genetic alteration in hepatocellular carcinoma is activation of the Wnt pathway, most often through somatic mutations that stabilize $\beta$-catenin. We and others have shown that human hepatocellular carcinomas that harbor activated Wnt pathway components are mutually exclusive with those that have a loss of Hippo signaling signature. ${ }^{31,32}$ This observation suggests that liver cancer can occur without Yap/ Taz activation and that these liver cancers constitute distinct molecular subtypes and most likely would be less responsive to targeted anti-Yap/Taz therapies. In addition, we propose that one mechanism that may contribute to adenoma formation that we observed in $y a p^{\Delta} /$ taz $^{\Delta}$ mutants might be via activation of the Wnt pathway. Additional experiments would be required to test this hypothesis.

In summary, we have shown that the Hippo signaling pathway coactivators Yap and Taz are not essential for achieving proper liver size during development or in the perinatal period but are required to mount an effective regenerative response following $\mathrm{PHx}$. Hence, molecular mechanisms that function to regulate liver size in embryos and in young adults remain undefined. Our results also show that Hippo signaling and Yap/Taz likely function in the context of other pro-regenerative programs that promote liver repair. Identification of these pathways and determining how they interface with Hippo signaling remains an important direction of future research in this area. Finally, although Yap and Taz are clearly potent inducers of hepatocellular carcinoma and frequently deregulated in liver diseases, our results demonstrate that Yap/Taz are not obligate oncogenes in the context of liver cancer. However, that we did not observe malignant hepatocellular carcinomas suggests that Yap and Taz may have a general role in liver cancer progression and that Yap/Taz-targeted therapies may be generally useful to inhibit tumor progression in the context of liver cancers. Additional studies will be required to address these and other important questions in the future.

\section{CONFLICT OF INTEREST}

The authors declare no conflict of interest.

\section{ACKNOWLEDGEMENTS}

The work is financially supported by DK120611.

\section{PUBLISHER'S NOTE}

Springer Nature remains neutral with regard to jurisdictional claims in published maps and institutional affiliations.

1 Penzo-Mendez Al, Stanger BZ. Organ-size regulation in mammals. Cold Spring Harb Perspect Biol 2015; 7: a019240.

2 Halder G, Johnson RL. Hippo signaling: growth control and beyond. Development 2011; 138: 9-22.
3 Pan D. The hippo signaling pathway in development and cancer. Dev Cell 2010; 19: 491-505.

4 Yu FX, Zhao B, Guan KL. Hippo pathway in organ size control, tissue homeostasis, and cancer. Cell 2015; 163: 811-828.

5 Camargo FD, Gokhale S, Johnnidis JB, Fu D, Bell GW, Jaenisch R et al. YAP1 increases organ size and expands undifferentiated progenitor cells. Curr Biol 2007; 17: 2054-2060.

6 Dong J, Feldmann G, Huang J, Wu S, Zhang N, Comerford SA et al. Elucidation of a universal size-control mechanism in Drosophila and mammals. Cell 2007; 130: 1120-1133.

7 Zhou D, Conrad C, Xia F, Park JS, Payer B, Yin Y et al. Mst1 and Mst2 maintain hepatocyte quiescence and suppress hepatocellular carcinoma development through inactivation of the Yap1 oncogene. Cancer Cell 2009; 16: 425-438.

8 Lu L, Li Y, Kim SM, Bossuyt W, Liu P, Qiu Q et al. Hippo signaling is a potent in vivo growth and tumor suppressor pathway in the mammalian liver. Proc Natl Acad Sci USA 2010; 107: 1437-1442.

9 Song H, Mak KK, Topol L, Yun K, Hu J, Garrett L et al. Mammalian Mst1 and Mst2 kinases play essential roles in organ size control and tumor suppression. Proc Natl Acad Sci USA 2010; 107: 1431-1436.

10 Lee KP, Lee JH, Kim TS, Kim TH, Park HD, Byun JS et al. The Hippo-Salvador pathway restrains hepatic oval cell proliferation, liver size, and liver tumorigenesis. Proc Natl Acad Sci USA 2010; 107: 8248-8253.

11 Heallen T, Zhang M, Wang J, Bonilla-Claudio M, Klysik E, Johnson RL et al. Hippo pathway inhibits Wnt signaling to restrain cardiomyocyte proliferation and heart size. Science 2011; 332: 458-461.

12 Halder G, Dupont S, Piccolo S. Transduction of mechanical and cytoskeletal cues by YAP and TAZ. Nat Rev Mol Cell Biol 2012; 13: 591-600.

13 Meng Z, Moroishi T, Guan KL. Mechanisms of Hippo pathway regulation. Genes Dev 2016; 30: 1-17.

14 Wang J, Xiao Y, Hsu CW, Martinez-Traverso IM, Zhang M, Bai Y et al. Yap and Taz play a crucial role in neural crest-derived craniofacial development. Development 2016; 143: 504-515.

15 Postic C, Magnuson MA. DNA excision in liver by an albumin-Cre transgene occurs progressively with age. Genesis 2000; 26: 149-150.

16 Mitchell $\mathrm{C}$, Willenbring $\mathrm{H}$. A reproducible and well-tolerated method for $2 / 3$ partial hepatectomy in mice. Nat Protoc 2008; 3: 1167-1170.

17 Grijalva JL, Huizenga M, Mueller K, Rodriguez S, Brazzo J, Camargo F et al. Dynamic alterations in Hippo signaling pathway and YAP activation during liver regeneration. Am J Physiol Gastrointest Liver Physiol 2014; 307: G196-G204.

18 Loforese G, Malinka T, Keogh A, Baier F, Simillion C, Montani M et al. Impaired liver regeneration in aged mice can be rescued by silencing Hippo core kinases MST1 and MST2. EMBO Mol Med 2017; 9: 46-60.

19 Zhang N, Bai H, David KK, Dong J, Zheng Y, Cai J et al. The Merlin/NF2 tumor suppressor functions through the YAP oncoprotein to regulate tissue homeostasis in mammals. Dev Cell 2010; 19: 27-38.

20 Lee DH, Park JO, Kim TS, Kim SK, Kim TH, Kim MC et al. LATS-YAP/TAZ controls lineage specification by regulating TGFbeta signaling and Hnf4alpha expression during liver development. Nat Commun 2016; 7: 11961.

21 Yi J, Lu L, Yanger K, Wang W, Sohn BH, Stanger BZ et al. Large tumor suppressor homologs 1 and 2 regulate mouse liver progenitor cell proliferation and maturation through antagonism of the coactivators YAP and TAZ. Hepatology 2016; 64: 1757-1772.

22 Septer S, Edwards G, Gunewardena S, Wolfe A, Li H, Daniel J et al. Yesassociated protein is involved in proliferation and differentiation during postnatal liver development. Am J Physiol Gastrointest Liver Physiol 2012; 302: G493-G503.

23 Bai H, Zhang N, Xu Y, Chen Q, Khan M, Potter JJ et al. Yes-associated protein regulates the hepatic response after bile duct ligation. Hepatology 2012; 56: 1097-1107.

24 Cai J, Zhang N, Zheng Y, de Wilde RF, Maitra A, Pan D. The Hippo signaling pathway restricts the oncogenic potential of an intestinal regeneration program. Genes Dev 2010; 24: 2383-2388.

25 Fan F, He Z, Kong LL, Chen Q, Yuan Q, Zhang S et al. Pharmacological targeting of kinases MST1 and MST2 augments tissue repair and regeneration. Sci Trans/ Med 2016; 8: 352ra108. 
26 Schlegelmilch K, Mohseni M, Kirak O, Pruszak J, Rodriguez JR, Zhou D et al. Yap1 acts downstream of alpha-catenin to control epidermal proliferation. Cell 2011; 144: 782-795.

27 Michalopoulos GK, DeFrances MC. Liver regeneration. Science 1997; 276: 60-66.

28 Machado MV, Michelotti GA, Pereira TA, Xie G, Premont R, Cortez-Pinto H et al. Accumulation of duct cells with activated YAP parallels fibrosis progression in non-alcoholic fatty liver disease. J Hepatol 2015; 63: 962-970.

29 Wang X, Zheng Z, Caviglia JM, Corey KE, Herfel TM, Cai B et al. Hepatocyte TAZNWWTR1 promotes inflammation and fibrosis in nonalcoholic steatohepatitis. Cell Metab 2016; 24: 848-862.

30 Liu-Chittenden Y, Huang B, Shim JS, Chen Q, Lee SJ, Anders RA et al. Genetic and pharmacological disruption of the TEAD-YAP complex suppresses the oncogenic activity of YAP. Genes Dev 2012; 26: 1300-1305.

31 Fitamant J, Kottakis F, Benhamouche S, Tian HS, Chuvin N, Parachoniak CA et al. YAP inhibition restores hepatocyte differentiation in advanced HCC, leading to tumor regression. Cell Rep 2015; 10 1692-1707.

32 Sohn BH, Shim JJ, Kim SB, Jang KY, Kim SM, Kim JH et al. Inactivation of Hippo pathway is significantly associated with poor prognosis in hepatocellular carcinoma. Clin Cancer Res 2016; 22: 1256-1264.

33 Valero V 3rd, Pawlik TM, Anders RA. Emerging role of Hpo signaling and YAP in hepatocellular carcinoma. J Hepatocell Carcinoma 2015; 2: 69-78.

(c) (i) $(-)$ This work is licensed under a Creative Commons Attribution-NonCommercial-NoDerivs

International License. The images or other third party material in this article are included in the article's Creative Commons license, unless indicated otherwise in the credit line; if the material is not included under the Creative Commons license, users will need to obtain permission from the license holder to reproduce the material. To view a copy of this license, visit http://creativecommons.org/licenses/by-nc-nd/4.0/

C) The Author(s) 2018 